\title{
EVALUATION OF MUSCLE QUALITY RELIABILITYAND RACIAL DIFFERENCES IN BODY COMPOSITION OF OVERWEIGHT INDIVIDUALS
}

\author{
Malia N. Melvin, Abbie E. Smith-Ryan, Hailee L. Wingfield, Sarah N. Fultz, and Erica J. \\ Roelofs \\ Applied Physiology Laboratory, Department of Exercise \& Sport Science, University of North \\ Carolina, Chapel Hill, North Carolina, USA
}

\begin{abstract}
The purpose of this study was to evaluate the reliability of ultrasound measures of muscle crosssectional area (mCSA) and echo intensity (EI) in overweight subjects. A secondary purpose was to evaluate racial differences in EI, mCSA and body composition. In 33 overweight subjects, mCSA and EI were determined from a panoramic ultrasound scan of the vastus lateralis. Body composition was determined using dual-energy X-ray absorptiometry (DXA). Reliability of mCSA and EI was calculated from the intra-class correlation coefficient (ICC), standard error of the measurement (SEM) and minimal difference (MD). The ICC, SEM and MD for mCSA and EI were $0.87,2.12,5.89$ and $0.74,4.58,12.69$, respectively. There were no significant racial differences in body composition ( $p>0.05$ ); however, EI was significantly lower for black subjects $(p=0.018)$. These results suggest ultrasound measures of mCSA and EI are reliable in overweight subjects, and EI may provide additional muscle composition evaluations, beyond DXA measures.
\end{abstract}

\section{Keywords}

Ultrasound; Echo intensity; Muscle cross-sectional area; Reliability; Overweight; Race

\section{INTRODUCTION}

\begin{abstract}
Measurements of muscle quality (MQ) have become increasingly popular as a non-invasive method for determining characteristics of strength, power and metabolic syndrome (Fukumoto et al. 2011; Goodpaster 2001). Muscle quality measurements allow for an enhanced analysis of muscle tissue through assessment of the amount of contractile versus non-contractile tissue within the muscle (Fukumoto et al. 2011), and are made largely using advanced and expensive technology, such as computed tomography (CT) and magnetic resonance imaging (MRI) (Goodpaster 2001; Katsiaras et al. 2005; Miljkovic et al. 2009; Tracy et al. 1999). MRI and CT technologies have commonly been used to quantify muscle cross-sectional area (mCSA) and, more recently, to evaluate intramuscular fat and
\end{abstract}

(C) 2014 World Federation for Ultrasound in Medicine \& Biology.

Address correspondence to: Abbie E. Smith-Ryan, Department of Exercise and Sport Science, University of North Carolina Chapel Hill, 209 Fetzer Hall, CB\# 8700, Chapel Hill, NC 27599-8700, USA. Abbsmith@email.unc.edu. 
connective tissue, as an estimate of muscle quality. These devices are expensive and use separate algorithms to quantify amounts of intramuscular soft tissue (Katsiaras et al. 2005; Miljkovic et al. 2009). Ultrasound (US) has more recently been correlated with MRI/CT for measures of mCSA (Ahtiainen et al. 2010; Mendis et al. 2010). Measures of intramuscular fat and connective tissue from full gross dissection, of an animal model, have also been correlated with gray-scale analysis of MQ from US scans (Pillen et al. 2009).

A major component of MQ when using US for measurement is echo intensity (EI). Echo intensity is a non-invasively acquired measurement that represents the amount of adipose and connective tissue infiltration within the muscle. As subcutaneous and skeletal muscle fat infiltration increases in overweight and obese individuals, a concomitant increase in EI and decrease in muscle quality are anticipated (Goodpaster et al. 2000; Miljkovic and Zmuda 2010; Nijboer-Oosterveld et al. 2011). Racial differences in MQ values have also been suggested. Previous studies using CT technology have found differences between black and white individuals in intramuscular fat (Miljkovic et al. 2009) and muscle density (Katsiaras et al. 2005). The relationship of race to EI has yet to be evaluated using US measures. Muscle size as measured by mCSA has been reported to be highly correlated with muscle function (English et al. 2012). Accurate assessments of mCSA may also be a useful tool to predict strength, power and overall health and quality of life.

Measurement of reliability has direct implications regarding the sensitivity and reproducibility of a measurement technique. As suggested by Weir (2005), common measures, such as intra-class correlation coefficient (ICC) and standard error of the measurement (SEM), represent the relative and absolute consistency of the measurements. ICCs, which are considered to be generalizable between laboratories and testers, account for random error when systematic error is trivial. Measures of reliability may be dependent on the variance of the individuals in the sample, and therefore, analysis of both ICC and SEM is necessary to reduce the likelihood of producing inflated reliability (Weir 2005). Furthermore, the reported reliability of a technique is vital in determining whether values from a single measurement are beyond the associated error. Reproducibility values also ensure that any intervention or change reported is in fact real, typically measured by a minimal difference (MD) value.

There are multiple factors including age, sex, race, training status and body composition that may yield varying results for mCSA and EI reliability. A recent meta-analysis provides evidence that US is a reliable method for measurement of mCSA in healthy subjects. Multiple studies have determined the reliability of US measures of mCSA of quadriceps muscles to be high (Ahtiainen et al. 2010; Bemben 2002; English et al. 2012; Mendis et al. 2010). However, studies investigating EI reliability have obtained mixed results. Minimal data exist regarding the reproducibility of mCSA and EI in overweight individuals. To the best of our knowledge, no study has investigated the reliability of mCSA and EI in overweight individuals as the primary focus of study. The purpose of this study was to investigate the reliability of US measures of mCSA and EI of the vastus lateralis (VL) in overweight subjects. A secondary purpose was to evaluate racial differences in EI, mCSA and body composition. 


\section{METHODS}

\section{Subjects}

Thirty-nine overweight (body mass index $>25 \mathrm{~kg} / \mathrm{m}^{2}$ ) individuals volunteered to participate in this study. Six were removed because of the inability to obtain a scan with clear and defined fascial borders of the VL, as a result of extremely large amounts of subcutaneous fat on the thigh. Therefore, 33 overweight men and women (mean \pm standard deviation [SD]age: $39.4 \pm 11.4 \mathrm{y}$, height: $174.1 \pm 10.0 \mathrm{~cm}$, weight: $94.2 \pm 15.1 \mathrm{~kg}$, percentage body fat: $32.4 \pm 7.1 \%)$ were evaluated in this study. Subjects were stratified by race: black $(\mathrm{n}=10)$ and white $(\mathrm{n}=23)$. Before testing, all subjects signed an informed consent form approved by the University's institutional review board for the protection of human subjects.

\section{Experimental design}

This study was completed in two visits; each visit consisted of the same body composition testing, completed by the same trained investigators. Subjects arrived at the laboratory after an 8-h fast. On arrival, height and weight were measured. Muscle cross-sectional area (mCSA) of the vastus VL was determined from a panoramic scan of the VL using B-mode ultrasound. Echo intensity was determined offline using ImageJ software (Version 1.37, National Institutes of Health, Bethesda, MD, USA). Subsequently, dual-energy X-ray absorptiometry (DXA) was used to determine body composition, and thigh fat thickness (THfat) was measured with a standard skinfold caliper.

\section{Ultrasound}

Muscle cross-sectional area and EI of the VL were determined using a GE Logiq-e B-mode ultrasound machine (GE Healthcare, Waukesha, WI, USA) from a panoramic scan at the midpoint of the thigh. The ultrasound settings (frequency: $10 \mathrm{~Hz}$, gain: 56, depth: $4.0 \mathrm{~cm}$ ) were kept constant to standardize mCSA and EI measures. A wide-band linear array ultrasound transducer (12 L-RS, 5-13 mHz, GE Healthcare) was held perpendicular to the tissue and swept across the skin at equal pressure from the lateral VL border to medial fascia separation (Fig. 1). The same technician performed each scan while the subject lay supine with the right leg extended and relaxed on the examination table. Muscle cross sectional area and EI were determined using Image J software in the standard histogram function, which uses gray-scale analysis of pixels ranging from 0 to 255 . Before measurement of mCSA and EI, each image was calibrated by measuring the number of pixels within a known distance of $1 \mathrm{~cm}$. As previously described by Cadore et al. (2012), in ImageJ, the same technician traced the outline of the VL for each patient's scan along the fascia border as close as possible to capture only the muscle to determine mCSA, and EI (Figs. 2, 3).

\section{Dual-energy X-ray absorptiometry}

Each subject completed a full body scan using the DXA (Apex Software Version 3.3, Hologic, Bedford, MA, USA) to determine lean mass (LM), fat mass (FM) and percentage body fat (\%fat). Each scan was performed by a trained DXA technician. Before testing, subjects were asked to remove all metal, thick clothing and heavy plastic to reduce interference with the scan. Age, height, weight and ethnicity were entered into the computer.

Ultrasound Med Biol. Author manuscript; available in PMC 2015 April 28. 
Subjects were placed supine in the center of the scanning table; if the participant's shoulders were too wide to fit in the area of the scan, thumbs were tucked under their buttocks to capture the full scan. Previous test-retest reliability in our lab on 10 individuals with a similar build were $\mathrm{ICC}=0.98, \mathrm{SEM}=0.85$ and coefficient of variation $(\mathrm{CV})=2.92 \%$ for fat mass; ICC $=0.99$, SEM $=1.07$ and $\mathrm{CV}=1.87 \%$ for lean mass; and $\mathrm{ICC}=0.98, \mathrm{SEM}=1.06$ and $\mathrm{CV}=3.14 \%$ for percentage fat.

\section{Thigh fat thickness}

A standard skinfold caliper (Lange, Cambridge, MA, USA) was used to measure subcutaneous adipose tissue of the thigh. THfat was determined as the average of two trial measurements taken within $3 \mathrm{~mm}$ of each other. The same technician performed each measurement at the midpoint of the right thigh while the subject stood upright with the majority of weight on the left leg, as described by the Anthropometric Standardization Reference Manual (Lohman et al. 1988).

\section{Statistical analysis}

In a custom-written Microsoft Excel spreadsheet (Mac 2011 Version 14.0.0, Microsoft, Redmond, WA, USA), one-way repeated-measures analyses of variance (ANOVAs) were used to evaluate the variability of mCSA and EI. Reliability of mCSA and EI was evaluated using the ICC, SEM, SEM as a percentage of the mean (SEM\%) and MD values. In SPSS Version 19 Statistical Analysis Software (IBM, Somers, NY, USA), a one-way ANOVA was used to evaluate racial comparisons of FM, LM, \% fat, EI, mCSA and THfat.

\section{RESULTS}

The reliability data for mCSA and EI are summarized in Tables 1 and 2 . When the data were stratified by race (Table 3$)$, there were no significant differences for FM $(p=0.144)$, LM $(p$ $=0.301), \%$ fat $(p=0.519)$ or mCSA $(p=0.841)$. However, EI was significantly lower $(\Delta=$ 7.8 arbitrary units [AU], $p=0.018)$ and THfat was significantly higher $(\Delta=17.3 \mathrm{~mm}, p<$ 0.001 ) for black than white patients.

\section{DISCUSSION}

The results of the present study indicate that use of B-mode US for measurement of mCSA and $\mathrm{EI}$ of the $\mathrm{VL}$ is reliable in overweight men and women. There were no racial differences for FM, LM, \%fat or mCSA; EI was significantly lower for black subjects $(45.7 \pm 10.3 \mathrm{AU}$, $p=0.018)$ than for white subjects $(53.5 \pm 7.2 \mathrm{AU})$. To our knowledge this is the first investigation in overweight individuals, reporting good reliability for mCSA and EI (ICC = $0.74-0.87 ; \mathrm{SEM}=2.12 \mathrm{~cm}^{2}$ and $4.58 \mathrm{AU}$, respectively).

Previous evaluations in healthy and aging populations have reported acceptable test-retest reliability for mCSA US measurements of quadriceps muscles, reporting ICC values of 0.72-0.99 and SEM values of $0.3-1.1 \mathrm{~cm}^{2}$ (Ahtiainen et al. 2010; Bemben 2002; Mendis et al. 2010). Bemben (2002) reported ICC values between 0.72 and 0.88 for mCSA of the rectus femoris in 133 healthy adults (age: $60.3 \pm 10.2 \mathrm{y}$ ), comparable to the reliability obtained in the present study (ICC $=0.87$ ) of the VL. It appears through calculated mean 
body mass index $\left(\mathrm{kg} / \mathrm{m}^{2}\right)$ that some individuals measured by Bemben (2002) were overweight; however, the entire sample was not categorized as overweight, nor could a separate analysis be run only on this population. Mendis et al. (2004) also reported values similar to those obtained in the present study: ICC and SEM values of 0.91 and $1.1 \mathrm{~cm}^{2}$ for the rectus femoris, 0.94 and $0.9 \mathrm{~cm}^{2}$ for the iliopsoas and 0.85 and $0.3 \mathrm{~cm}^{2}$ for the sartorius in nine healthy adults. A more recent study by Ahtiainen et al. (2010) reported a greater ICC value (0.997) for the VL in 27 healthy males and a smaller SEM of $0.38 \mathrm{~cm}^{2}$. It is likely that a healthy population will have results of higher reliability because of high-quality muscle with less fat and connective tissue infiltration, allowing for a more clearly defined fascial border for tracing and analysis of mCSA and EI. Muscle CSA of the quadriceps femoris muscles has also been found to be highly reliable in children (ICC $=0.99)$ (Kanehisa et al. 1994), obese adults with rheumatoid arthritis and healthy obese controls (\%fat $=38.5 \pm$ $2.2 \%)($ ICC $=0.81-0.95)$ (Matschke et al. 2010). The numeric value of EI is determined by a gray-scale analysis of an US image and has previously been reported to be an indirect measure of strength, insulin insensitivity and the development of metabolic syndrome (Goodpaster 2001; Katsiaras et al. 2005; Miljkovic et al. 2009). The possible relationship between EI and insulin insensitivity suggests US measures of EI may provide a relatively quick and inexpensive method to predict individuals who may be at a higher risk for developing metabolic syndrome. Previous literature also suggests the use of EI to determine changes in strength, power, cardiovascular function and exercise performance in young and old individuals (Achten and Jeukendrup 2004; Cadore et al. 2012; Fukumoto et al. 2011). Cardiorespiratory fitness has been found to be associated with overall functional capacity; therefore, it may suggest that US measures could be a useful method to predict the overall functional capacity of individuals with greater health risks because of obesity, age and physical inactivity.

Previous studies have reported EI reliability values from US measurements with a wide disparity (ICC $=0.209-0.99$ ) in various populations, such as overweight elderly women (Fukumoto et al. 2011), healthy middle-aged women (Fukumoto et al. 2012) and young individuals (Strasser et al. 2013). Fukumoto et al. (2011) reported high reliability in the rectus femoris $(\mathrm{ICC}=0.91$ ) for seven overweight elderly women. Test-retest reliability analysis on seven healthy women (age: $57.7 \pm 6.4$ ) also reported high ICC values of the gluteus maximus and medius, as well as abdominal muscles (ICC $=0.87-0.99)$ (Fukumoto et al. 2012). Although reliability data reported by Fukumoto et al. (2012) were found to be higher than reported in the present study (ICC $=0.74)$, Strasser et al. (2013) observed low (ICC $=0.209)$ to moderate (ICC $=0.65)$ reliability of $\mathrm{EI}$ in the quadriceps muscles in 52 young (age: $24.2 \pm 3.7 \mathrm{y}$, ICC for VL $=62.3$ ) and elderly (age: $67.8 \pm 4.8 \mathrm{y}, \mathrm{ICC}$ for VL $=$ $29.7)$ individuals. Variance in the reliability values may be a result of differences in the technique used to obtain the US scan, including probe position and pressure, as well as the US settings for depth, frequency and gain. To make more consistent comparisons across studies, the same US equipment and settings may be valuable. As EI is measured by the average pixel values between 0 (black) and 255 (white), it is essential the images be of the same quality to estimate valid EI measures across studies. The quality of the image may be influenced by shadows occurring during a panoramic scan as a result of not keeping the probe flush against the skin. Special notice should be taken of the clarity of the scan when 
measuring muscle quality; if the probe loses contact with the skin, gaps are estimated from the US software and introduce error. To minimize error and inter-rater variability, in the present study, the same technician performed all of the US scans, and the same technician performed all of the mCSA and EI analyses in ImageJ software.

To our knowledge, no previous published study has evaluated racial reliability comparisons of mCSA and EI US measures. Possible differences in EI between races may be observed because of muscle architecture differences including pennation angle, fiber type composition and muscle density. Ama et al. (1986) detected muscle fiber differences between sedentary black and white males, with white individuals reporting a higher type I fiber ratio. For $\mathrm{mCSA}$, the present study determined high reliability in both black $(\mathrm{ICC}=0.964, \mathrm{SEM}=$ $\left.1.66 \mathrm{~cm}^{2}\right)$ and white $\left(\mathrm{ICC}=0.810, \mathrm{SEM}=2.28 \mathrm{~cm}^{2}\right)$ subjects; however, reliability appears to be higher in black individuals. For EI, the present study determined high reliability for black subjects $(\mathrm{ICC}=0.923, \mathrm{SEM}=3.93 \mathrm{AU})$ and white subjects $(\mathrm{ICC}=0.940, \mathrm{SEM}=4.03$ AU). Evaluation of overall racial differences revealed no significant differences in mCSA or any body composition variables. Echo intensity was the only measure that resulted in a significant difference between black and white cohorts $(p=0.018, \Delta=7.8 \mathrm{AU})$. There is limited research regarding differences in race that may explain why higher-density muscle was found in black (EI $=45.7 \pm 10.3 \mathrm{AU})$ compared with white $(\mathrm{EI}=53.5 \pm 7.2 \mathrm{AU})$ subjects. Abe et al. (1999) found no significant differences in pennation angle, fascicle length and muscle thickness between white and black collegiate football players using US technology. Miljkovic et al. (2009) and Katsiaras et al. (2005), using CT scans, found greater intramuscular adipose tissue in black males than in white males; Katsiaras et al. (2005) also reported greater muscle density values in black elderly males. Therefore, further research may be warranted to investigate differences in fiber type composition, pennation angle and muscle density between races to gain a better understanding of EI differences.

As previously mentioned, body composition variables measured by DXA, such as \%fat, LM and FM, were not capable of detecting differences between racial groups. Thigh skinfold fat thickness, however, did detect racial differences; black subjects (THfat $=59.9 \pm 6.9 \mathrm{~mm}$ ) had greater amounts of subcutaneous fat compared with white subjects (THfat $=42.6 \pm 13.0$ $\mathrm{mm})(p<0.001)$. The THfat values are in opposition to the EI results, suggesting a greater muscle quality in black versus white individuals. Katsiaras et al. (2005), who also measured racial differences from subcutaneous fat and muscle quality and found greater subcutaneous fat $\left(\mathrm{SF}=117.0 \pm 46.4 \mathrm{~cm}^{2}\right)$, greater quadriceps area $\left(\mathrm{QA}=46.2 \pm 8.7 \mathrm{~cm}^{2}\right)$, but lower quadriceps density $(\mathrm{QD}=38.4 \pm 6.1 \mathrm{HU})$, in black elderly women compared with white women $\left(\mathrm{SF}=95.8 \pm 36.5 \mathrm{~cm}^{2}, \mathrm{QA}=39.8 \pm 6.4 \mathrm{~cm}^{2}, \mathrm{QD}=40.5 \pm 6.0 \mathrm{HU}\right)$. The previous racial differences reported between SF, QA and QD do not follow the same relationship reported in the present study, which could be a result of a larger sample size $(n=1512)$, muscle quality analysis differences between CT and US or age differences ( $75.5 \pm 2.8 \mathrm{y}$ vs. $39.4 \pm 11.4 \mathrm{y}$ in the present study). There may be a significant relationship between subcutaneous thigh fat and muscle quality and race; additional data are needed for more clear associations.

Limitations within the present study include the US depth setting, which was not sufficient for all subjects to observe the entire muscle and, therefore, varied across subjects. As the 
present study was part of a larger study, the subjects whose entire muscle was not observed because of greater amounts of subcutaneous fat were not included in the current sample $(n=$ 33) for mCSA and EI analysis. Another consideration for EI measurements is that EI does not differentiate between intramuscular fat and connective tissue, and therefore it cannot be stated from this study that low muscle quality is due to increased intramuscular fat, but from both fat and connective tissue. Previous studies using computed tomography (Katsiaras et al. 2005; Miljkovic et al. 2009) have observed that black individuals have greater intramuscular fat compared with white individuals. Future invasive comparisons (i.e., muscle biopsy vs. US) are suggested to quantify connective tissue and US EI estimations.

\section{CONCLUSIONS}

Ultrasound is a reliable tool for measuring mCSA and EI in overweight black and white individuals. There were no significant differences in mCSA or DXA body composition measurements between the black and white overweight individuals, but there was a significant difference in EI. Lower EI in overweight black individuals may suggest that black individuals have greater muscle quality (i.e., decreased intramuscular adipose and connective tissue, decreased pennation angle and increased muscle density) in comparison to overweight white individuals. On the basis of these initial findings, EI measured from a Bmode US may be useful in identifying muscle quality and/or racial differences, undetected by general body composition measures. US is a portable, relatively inexpensive tool that is readily available in most clinical settings. Therefore, US muscle quality measures may be beneficial in predicting individuals who are predisposed to metabolic or functional capacity concerns because of various issues related to obesity and increased intramuscular fat. Measures of mCSA may also be useful in determining the effects of a variety of interventions and conditions (English et al. 2012; Tracy et al. 1999), such as resistance training, surgery and cancer, to detect changes in muscle integrity for at-risk individuals.

\section{Acknowledgments}

This study was supported by funding from the Nutrition Obesity Research Center (P30 DK056350).

\section{References}

Achten J, Jeukendrup AE. Optimizing fat oxidation through exercise and diet. Nutrition. 2004; 20:716-727. [PubMed: 15212756]

Ahtiainen JP, Hoffren M, Hulmi JJ, Pietikäinen M, Mero AA, Avela J, Häkkinen K. Panoramic ultrasonography is a valid method to measure changes in skeletal muscle cross-sectional area. Eur J Appl Physiol. 2010; 108:273-279. [PubMed: 19777252]

Abe T, Brown JB, Brechue WF. Architectural characteristics of muscle in black and white college football players. Med Sci Sports Exercise. 1999; 31:1448-1452.

Ama PFM, Simoneau JA, Boulay MR, et al. Skeletal muscle characteristics in sedentary black and Caucasian males. J Appl Physiol. 1986; 61:1758-1761. [PubMed: 2946652]

Bemben MG. Use of diagnostic ultrasound for assessing muscle size. J Strength Conditioning Res. 2002; 16:103-108.

Cadore EL, Izquierdo M, Conceição M, Radaelli R, Pinto RS, Baroni BM, Kruel LFM. Echo intensity is associated with skeletal muscle power and cardiovascular performance in elderly men. Exp Gerontol. 2012; 47:473-478. [PubMed: 22525196] 
English C, Fisher L, Thoirs K. Reliability of real-time ultrasound for measuring skeletal muscle size in human limbs in vivo: A systematic review. Clin Rehab. 2012; 26:934-944.

Fukumoto Y, Ikezoe T, Tateuchi H, Tsukagoshi R, Akiyama H, So K, Ichihashi N. Muscle mass and composition of the hip, thigh and abdominal muscles in women with and without hip osteoarthritis. Ultrasound Med Biol. 2012; 38:1540-1545. [PubMed: 22749818]

Fukumoto Y, Ikezoe T, Yamada Y, Tsukagoshi R, Nakamura M, Mori N, Ichihashi N. Skeletal muscle quality assessed from echo intensity is associated with muscle strength of middle-aged and elderly persons. Eur J Appl Physiol. 2011; 112:1519-1525. [PubMed: 21847576]

Goodpaster BH. Skeletal muscle lipid content and insulin resistance: Evidence for a paradox in endurance-trained athletes. J Clin Endocrinol Metab. 2001; 86:5755-5761. [PubMed: 11739435]

Goodpaster BH, Theriault R, Watkins SC, Kelley DE. Intramuscular lipid content is increased in obesity and decreased by weight loss. Metab Clin Exp. 2000; 49:467-472. [PubMed: 10778870]

Kanehisa H, Ikegawa S, Tsunoda N, Fukunaga T. Strength and cross-sectional area of knee extensor muscles in children. Eur J Appl Physiol Occup Physiol. 1994; 68:402-405. [PubMed: 8076619]

Katsiaras A, Newman AB, Kriska A, Brach J, Krishnaswami S, Feingold E, Goodpaster BH. Skeletal muscle fatigue, strength, and quality in the elderly: The Health ABC Study. J Appl Physiol. 2005; 99:210-216. [PubMed: 15718402]

Lohman, TG.; Roche, AF.; Martorell, R. Anthropometric standardization reference manual. Champaign, IL: Human Kinetics; 1988. p. 177

Matschke V, Murphy P, Lemmey AB, Maddison P, Thom JM. Skeletal muscle properties in rheumatoid arthritis patients. Med Sci Sports Exercise. 2010; 42:2149-2155.

Mendis MD, Wilson SJ, Stanton W, Hides JA. Validity of real-time ultrasound imaging to measure anterior hip muscle size: A comparison with magnetic resonance imaging. J Orthop Sports Phys Ther. 2010; 40:577-581. [PubMed: 20479536]

Miljkovic I, Cauley JA, Petit MA, Ensrud KE, Strotmeyer E, Sheu Y, Zmuda JM. Greater adipose tissue infiltration in skeletal muscle among older men of African ancestry. J Clin Endocrinol Metabol. 2009; 94:2735-2742.

Miljkovic I, Zmuda JM. Epidemiology of myosteatosis. Curr Opin Clin Nutr Metab Care. 2010; 13:260-264. [PubMed: 20179586]

Nijboer-Oosterveld J, Van Alfen N, Pillen S. New nomal values for quantitative muscle ultrasound: Obesity increases muscle echo intensity. Muscle Nerve. 2011; 43:142-143. [PubMed: 21082696]

Pillen S, Tak R, Zwarts M, Lammens M, Verrijp K, Arts I, Van Der Laak J, Hoogerbrugge P, Van Engelen B, Verrips A. Skeletal muscle ultrasound: correlation between fibrous tissue and echo intensity. Ultrasound Med Biol. 2009; 35:443-446. [PubMed: 19081667]

Strasser EM, Draskovits T, Praschak M, Quittan M, Graf A. Association between ultrasound measurements of muscle thickness, pennation angle, echogenicity and skeletal muscle strength in the elderly. Age. 2013; 35:2377-2388. [PubMed: 23456136]

Tracy BL, Ivey FM, Hurlbut D, Martel GF, Lemmer JT, Siegel EL, Hurley BF. Muscle quality: II. Effects of strength training in 65-to 75-yr-old men and women. J Appl Physiol. 1999; 86:195-201. [PubMed: 9887131]

Weir J. Quantifying test-retest reliability using the intra-class correlation coefficient and the SEM. J Strength Conditioning Res. 2005; 19:231-240. 


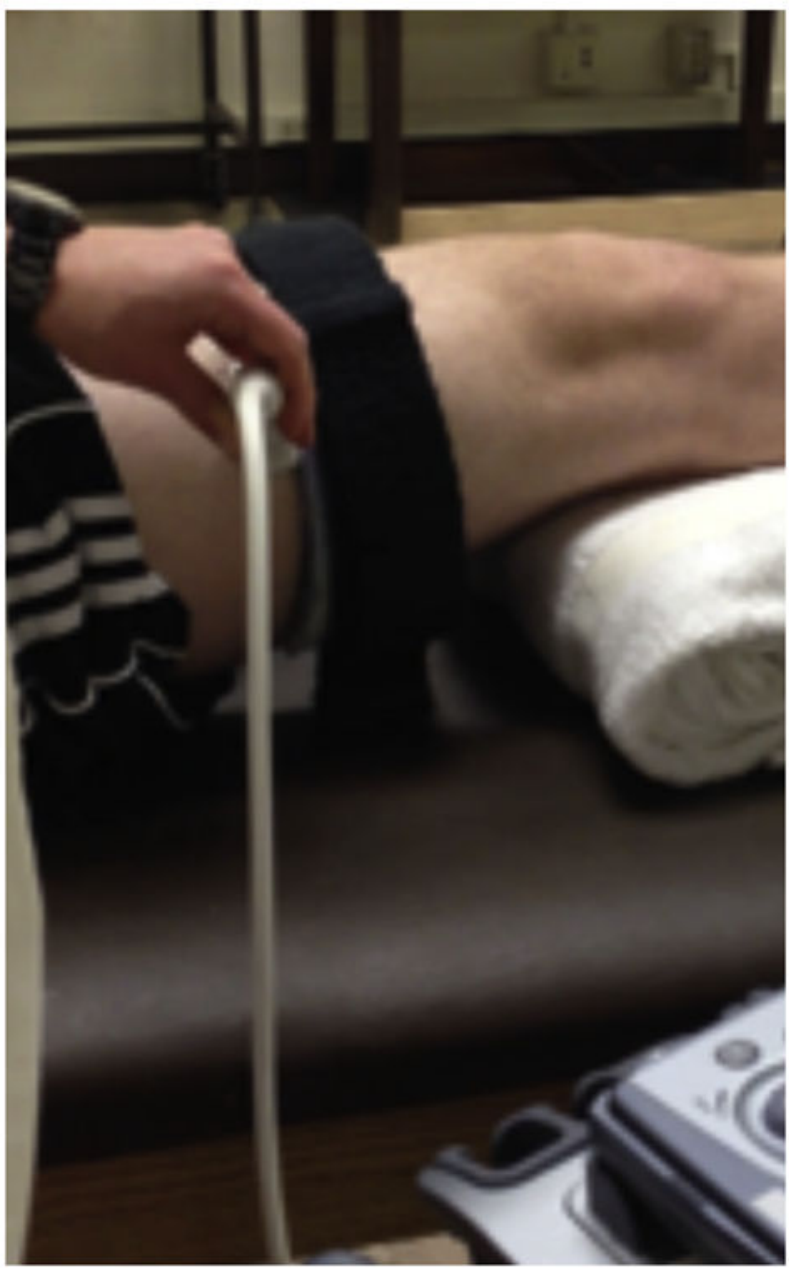

Fig. 1.

Image of B-mode ultrasound scan of the vastus lateralis using $12 \mathrm{~L}-\mathrm{RS}$ transducer probe held perpendicular to the tissue. 


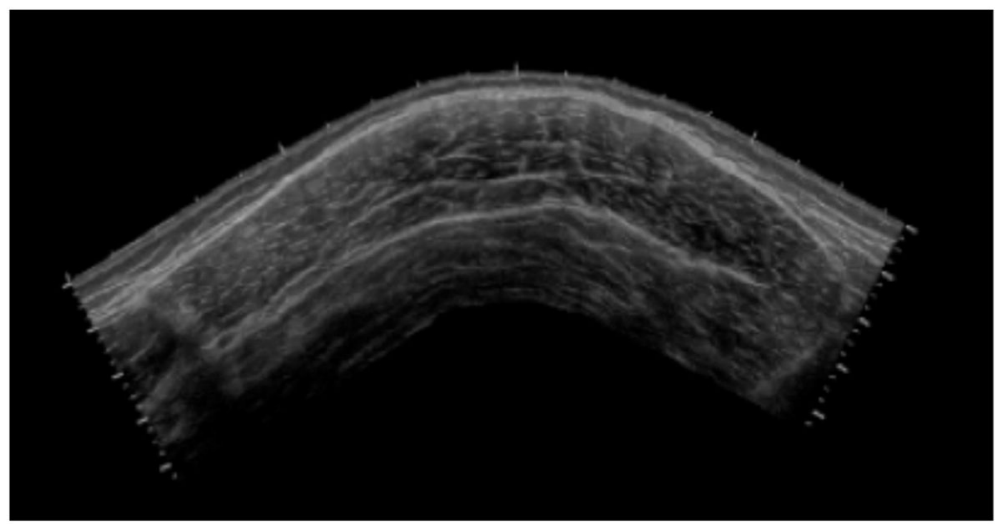

Fig. 2.

Ultrasound image of a panoramic scan of the vastus lateralis. 


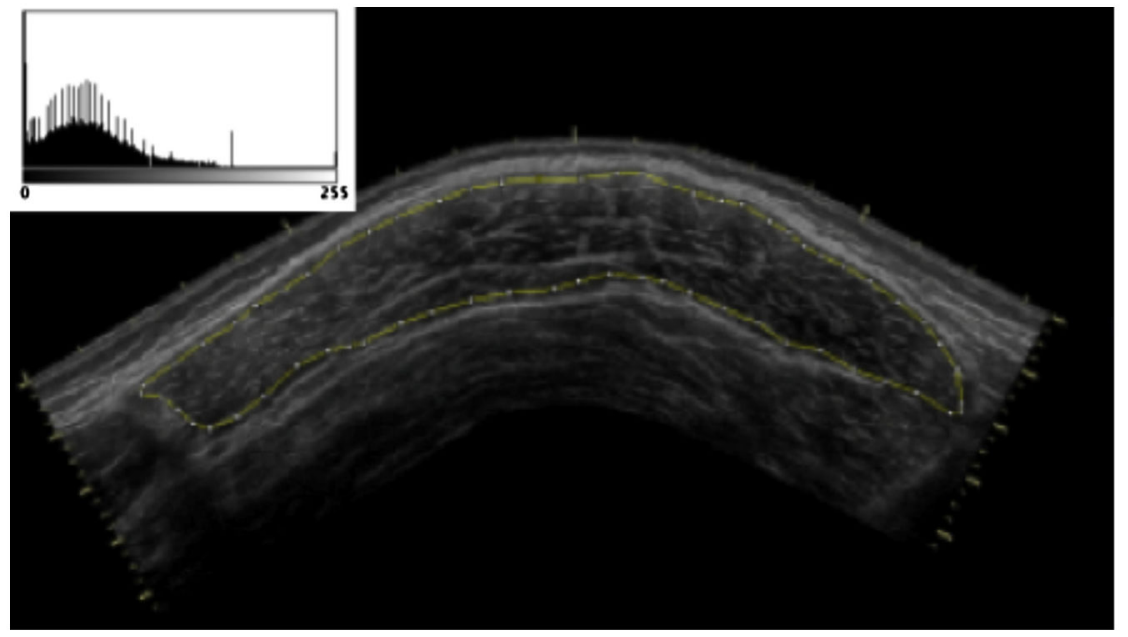

Fig. 3.

ImageJ software outline and histogram of the vastus lateralis to determine muscle crosssectional area and echo intensity. 


\section{Table 1}

Reliability statistics for EI and mCSA of the vastus lateralis

\begin{tabular}{lcccr}
\hline & ICC $_{(\mathbf{2}, \mathbf{1})}$ & SEM & SEM\% & Minimal difference \\
\hline mCSA $\left(\mathrm{cm}^{2}\right)$ & 0.87 & 2.12 & 13.14 & 5.89 \\
$\mathrm{EI}(\mathrm{AU})$ & 0.74 & 4.58 & 10.04 & 12.69 \\
\hline
\end{tabular}

$\mathrm{EI}=$ echo intensity; $\mathrm{mCSA}=$ muscle cross-sectional area; ICC =intra-class coefficient; $\mathrm{SEM}=$ standard error of the measurement; \%SEM = SEM as a percentage of the mean; $\mathrm{AU}=$ arbitrary units. 
Table 2

Comparisons of reliability statistics for EI and mCSA of the vastus lateralis by race

\begin{tabular}{|c|c|c|c|c|}
\hline & $\operatorname{ICC}_{(2,1)}$ & SEM & SEM\% & Minimal difference \\
\hline \multicolumn{5}{|c|}{$\mathrm{mCSA}\left(\mathrm{cm}^{2}\right)$} \\
\hline Black & 0.964 & 1.66 & 9.89 & 4.60 \\
\hline White & 0.810 & 2.28 & 11.05 & 5.02 \\
\hline \multicolumn{5}{|l|}{ EI (AU) } \\
\hline Black & 0.923 & 3.93 & 8.87 & 10.90 \\
\hline White & 0.940 & 4.03 & 7.64 & 11.17 \\
\hline
\end{tabular}

$\mathrm{EI}=$ echo intensity; $\mathrm{mCSA}=$ muscle cross-sectional area; ICC =intra-class coefficient; SEM =standard error of the measurement; \%SEM = SEM as a percentage of the mean; $\mathrm{AU}=$ arbitrary units. 


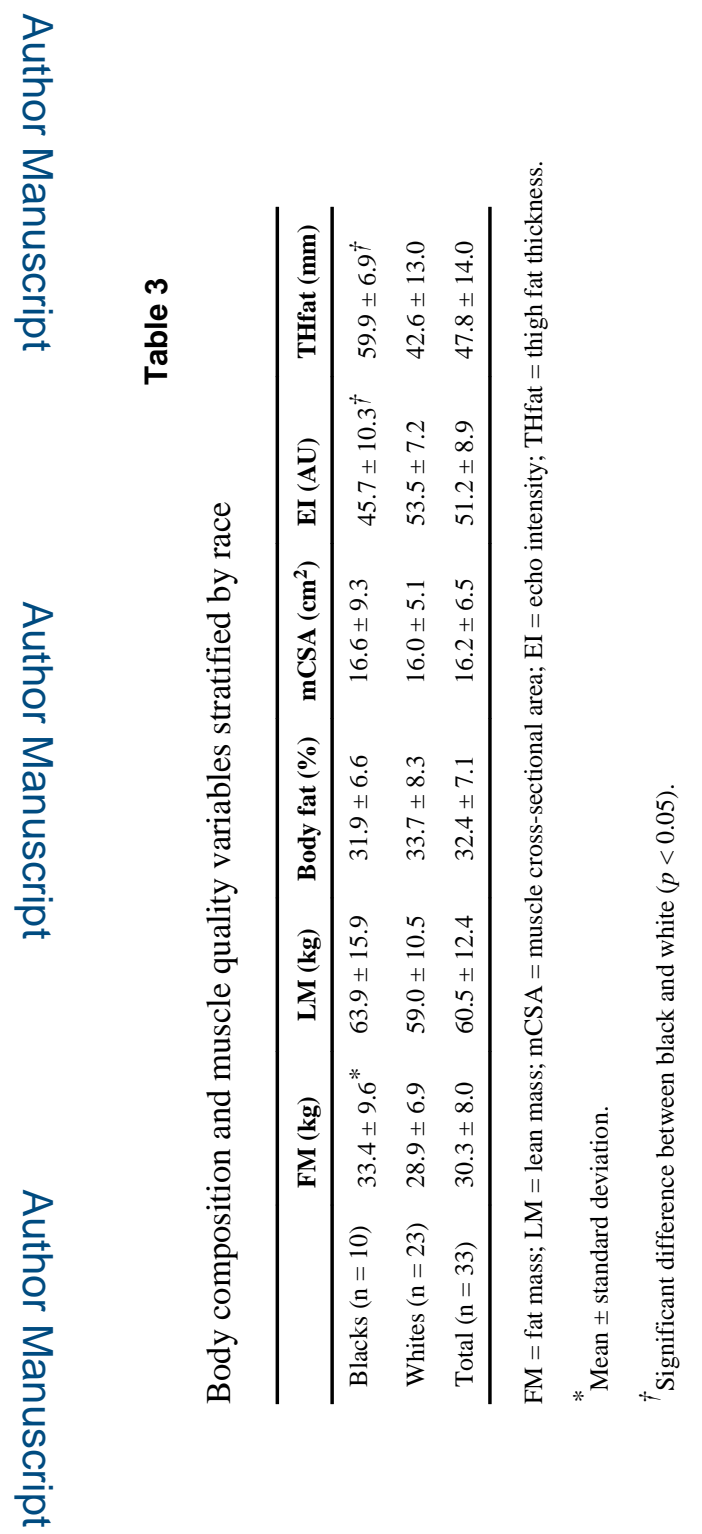

